\title{
Bioéconomie : un retour historique sur deux problématisations de l'usage des ressources renouvelables
}

\author{
Nicolas Béfort ${ }^{1, *}$ et Martino Nieddu ${ }^{2, a}$ \\ 1 Économie, NEOMA Business School, Chaire Bioéconomie et Développement Soutenable, Reims, France \\ 2 Économie, Laboratoire REGARDS, Université de Reims Champagne-Ardenne, Reims, France
}

Reçu le 12 mars 2018. Accepté le 7 décembre 2019

La bioéconomie, telle qu'elle est portée institutionnellement par l'OCDE, correspond à une certaine vision du futur. Il s'agit d'un élément crucial de coordination des acteurs scientifiques et industriels confrontés à l'incertitude. Pour interroger cette construction de l'avenir, les auteurs se livrent à ce qu'on pourrait appeler un « retour vers le futur»: ils se penchent sur deux moments passés de problématisation du futur de l'usage des ressources renouvelables qu'ont constitué la chemurgy dans les années 1920-1930 et la bioindustrie dans les années 1960-1970 (voir aussi Vivien, NSS, 2019, 27, 2 pour cette dernière prônée par Joël de Rosnay). Ils montrent ainsi la grande permanence des dispositifs de qualification et de coordination, des problématiques productives sous-jacentes et des ressources mobilisées. Cela témoigne, de la part des acteurs dominants, de la mobilisation de patrimoines productifs collectifs afin de préempter régulièrement le futur, ce qui fait écho à ce qui avait été mis en évidence dans le domaine de la chimie verte par Nieddu et al. (NSS, 2014, 22, 2).

La Rédaction

\begin{abstract}
Résumé - La définition d'une bioéconomie fondée sur l'usage des ressources renouvelables passe aujourd'hui par un intense travail institutionnel de construction de «visions du futur». Elles portent sur la problématisation des formes de l'industrie envisagée, de ses rapports au «reste du monde» et sur la régulation du changement. Elles sont des grands récits à vocation performative portant sur les promesses technico-économiques et la nécessité de participer à d'inévitables ruptures schumpétériennes. Une façon de les discuter est d'explorer dans l'Histoire des moments identiques. Nous en avons retenu deux : (1) Les années 1920-1930 aux États-Unis, où certains acteurs s'allient pour donner naissance à une chemurgy créant des produits industriels sur substrat agricole. (2) Les années 1976-1980, où la crise de la chimie à base de pétrole conduit à envisager un renouveau à partir d'une chimie des sucres.
\end{abstract}

Mots clés : agriculture / ressources naturelles / dispositifs institutionnels / bioéconomie

\begin{abstract}
The bioeconomy: a historical review of two problematizations of the use of renewable resources. The bioeconomy is becoming increasingly important in public policy, especially in the European Union and the USA. It is described either as a new biotechnological industrial revolution, or as a great transition toward the use of renewable resources, both of these through the development of 'biorefineries'. To organize the transition, agro-industry, and the chemical, wood and biotechnology industries are involved in intensive institutional work. This work aims to problematize the organization of this new industrial bioeconomy, its links with other sectors, and the regulation of the change. To do so, public policies support backcasting exercises and roadmap development. However, the history of this transition toward the use of renewable resources shows that similar problematizations were conducted in the past. We look at two historical periods. First, very similar promises to those of today were formulated in the 1930s, by the USA chemurgy movement. Second, during the 1980s, the chemical industry became interested in sugar
\end{abstract}

\footnotetext{
*Auteur correspondant : nicolas.befort@neoma-bs.fr

${ }^{a}$ Martino Nieddu nous a quittés brutalement durant le processus d'édition de ce texte. Cet article, issu de nos nombreux échanges, lui est dédié.
} 
following the oil crisis of the 1970s. During this period the problematization of the biorefinery as a structuring artefact emerged. In this paper, we review these two historical periods to interpret today's 'visions of the future' from a historical perspective. We conclude this study by comparing and contrasting former and current tensions.

Keywords: agriculture / natural resources / institutions / bioeconomy

\section{De la bioéconomie d'aujourd'hui à celles du passé}

Dans son document ${ }^{1}$ de 2012, la Commission européenne définissait la bioéconomie comme englobant «la production de ressources biologiques renouvelables et la transformation de ces ressources et des flux de déchets en produits à valeur ajoutée comme des denrées alimentaires, des aliments pour animaux, des bioproduits et de la bioénergie ». Elle donnait ainsi l'impulsion à un vaste mouvement de réflexion, prit la forme de l'élaboration de stratégies nationales vers la bioéconomie (Staffas et al., 2013; Lokko et al., 2018), lesquelles réincorporaient des exercices antérieurs de backcasting portant sur la bioraffinerie. Il s'agit donc de travaux de problématisation d'une industrie (Jullien et Smith, 2012), compris ici comme un effort de légitimation de la constitution d'un espace méso-économique particulier.

Or, ce questionnement n'est pas nouveau. Il est donc intéressant de situer les discours de nouveauté technologique radicale, tenus aujourd'hui, à l'aune d'autres moments historiques où la discussion sur la problématisation d'une industrie issue du renouvelable a été importante. Au cours de nos divers travaux, nous en avons identifié deux qui auraient pu être des moments de développement d'une bioéconomie.

Aux États-Unis, les années 1920 ont été un moment de cristallisation durant lequel s'est formé un bloc social à l'initiative d'isolationnistes et d'agrairiens qui rejoignent le projet de scientifiques pionniers, cherchant à sortir le Sud profond de la misère et de la monoculture du coton par l'usage non alimentaire d'autres produits et de coproduits de l'agriculture. Ils cherchent à théoriser et à promouvoir ce qu'ils avaient baptisé chemurgy. On s'appuie pour ce faire sur des travaux d'historiens des techniques (Finlay, 1997; 2003) ou des politiques économiques (Pursell, 1969), la très abondante documentation historique réunie par le centre américain d'information sur le soja (Shurtleff et Aoyagi, 2011) ainsi que sur la somme de documents de l'American Chemistry Society.

À la fin des années 1970, la crise de la chimie, liée au choc pétrolier, à la saturation de ses marchés et au

\footnotetext{
${ }^{1}$ Ce document prolonge la stratégie de Lisbonne adoptée en 2000. Elle lançait la knowledge-based economy (KBE), qui sera déclinée en une knowledge-based bio-economy (KBBE) en 2007.
}

ralentissement de ses innovations, conduit à explorer la chimie des sucres comme vecteur de nouvelle croissance. Lors des exercices de prospective de l'époque (publiés entre 1979 et 1982), une grande partie des voies explorées aujourd'hui sont mentionnées, d'où notre intérêt pour ce moment particulier de problématisation. Les biotechnologies sont alors «élues » comme l'un des deux domaines par lesquels un retour de la croissance est attendu. On s'appuiera ici sur les travaux de l'OCDE de la fin des années 1970 ainsi que sur les numéros spéciaux de la Revue d'économie industrielle (Chesnais, 1981) et de Futuribles (Cohendet, 1982) sur la crise de la chimie en Europe et ses perspectives de rebond, à partir des biotechnologies, de la biomasse et de la notion de bioindustries.

Nous suivrons le plan d'exposé suivant: dans une première partie, nous présenterons le cadre théorique mobilisé pour rendre compte de la façon dont les décisions des acteurs au moment où ils les prennent reposent sur la construction de visions du futur, à partir des concepts de promesses technico-économiques et de patrimoines productifs collectifs. Dans une deuxième partie, nous exposerons le cas de la bioéconomie contemporaine et montrerons quels sont ses enjeux vis-à-vis de la perspective adoptée. Dans une troisième partie, nous prendrons le cas de la chemurgy en la reliant aux promesses technico-économiques actuelles. Dans une quatrième partie, nous ferons de même pour le moment d'orientation vers la «bioindustrie» au début des années 1980. Nous discuterons dans la conclusion la portée de ces investigations ${ }^{2}$.

\section{Problématisation, visions du futur et promesses}

\section{Les visions du futur comme fondement de la problématisation des activités économiques}

La socioéconomie souligne le rôle clé des espérances dans l'innovation et le fonctionnement économique (Borup et al., 2006; Beckert, 2013 ; 2016). Les visions du futur ainsi définies sont rendues nécessaires par le

\footnotetext{
${ }^{2}$ Ce travail a été soutenu par la chaire Bioéconomie et Développement soutenable de Neoma Business School qui est financée par la Communauté urbaine du Grand Reims, la CCI Marne en Champagne, la Fondation du Site Paris-Reims et Neoma Business School.
} 
besoin qu'ont les acteurs de stabiliser leur environnement en raison de l'incertitude radicale du monde économique (White et al., 2008). Ceux-ci produisent des «fictions» fondées sur des outils spécifiques de calculs et d'anticipation du futur. Le futur devient ainsi un enjeu de représentations à même de guider les décisions d'allocations de ressources actuelles. Les formes de prédiction (modélisations, taux de croissance, etc.) et d'anticipation du futur ne sont pas contestées dans un environnement stabilisé, mais elles le sont en période de crises (Boyer, 2018). En mobilisant des visions du futur, les acteurs agissent «comme si» le futur existait, participant à créer un futur présent, et, en raison de l'existence de visions du futur concurrentes, ils contribuent à dessiner un futur potentiellement différent de leur vision.

Néanmoins, toutes les espérances n'ont pas cette capacité de mise en mouvement par l'allocation de ressources (institutions, connaissances, investissements, etc.) pour atteindre ce futur espéré. Les promesses technico-économiques ont cette capacité, si elles sont reconnues comme (i) légitimes pour répondre à un problème social et (ii) crédibles dans leur réponse (Joly, 2010).

(i) La définition du problème social posé et la réponse apportée relèvent de stratégies de problématisation (Jullien et Smith, 2012). Par exemple, les biotechnologies et la biologie de synthèse se construisent grâce à un arsenal de promesses revendiquant une transformation efficiente des ressources renouvelables permettant de résoudre de grands problèmes mondiaux (faim, changement climatique, santé, etc.) [Vivien et al., 2019]. Néanmoins, la reconnaissance de la légitimité de la promesse passe par la production de vérités, de preuves ou de modèles. Dans le cas des agromatériaux, les feuilles de route ou les grands états de l'art (reviews) jouent ce rôle particulier de construction partagée du futur dans un espace d'exploration technologique (Nieddu, 2013). Qu'il s'agisse de publications académiques, d'études de marchés ou de rapports d'institutions, ces documents sont des «cartes $\operatorname{cognitives}^{3}$ » réalisant le recensement ordonné d'une grappe d'opportunités technico-économiques en vue de légitimer la promesse formulée sur une trajectoire technologique. Il s'agit en réalité d'exercices non de prospective, au sens de projection dans le futur, mais de backcasting ou de rétropolation (Nieddu et al., 2014). À la différence de la méthode des scénarios qui part du présent pour identifier des futurs possibles, le backcasting part d'une vision du futur pour identifier le chemin de transition à suivre à partir de maintenant et les verrous technico-économiques à lever en cours de route (Langeveld et al., 2010).

\footnotetext{
${ }^{3}$ C'est-à-dire des représentations partagées de cet espace de concurrence.
}

(ii) La crédibilité des promesses est évaluée et doit aussi être reconnue. La composition des consortiums d'acteurs et de leurs activités passées est garante de cette crédibilité (Joly, 2010). Transcrites dans des artefacts, des routines ou des normes, les promesses sont donc productrices d'irréversibilité.

\section{Passé, présent et futur: le rôle des patrimoines productifs collectifs}

Les promesses technico-économiques prennent la forme d'engagements par l'allocation de ressources. Ces engagements sont pris dans des cycles d'espérances et de désappointements (Borup et al., 2006). Comment les acteurs résistent-ils à ces périodes de désappointement ? Les promesses sont liées à des activités présentes et passées de production ayant à la fois des formes tangibles (des brevets, des connaissances, des objets démonstratifs, etc.) et intangibles (des représentations). Les acteurs cherchent à préserver ces ressources dans des espaces spécifiques. C'est en raison de cette logique de préservation et de projection dans le futur que nous qualifions ces ressources mobilisées pour la formation de promesses technico-économiques de patrimoines productifs collectifs (Nieddu et al., 2010; 2014).

$\mathrm{Du}$ point de vue théorique, les patrimoines sont constitués par des ressources qui : (1) sont recherchées pour leur valeur collective, car elles permettent la coordination des acteurs dans la division du travail; (2) doivent être partagées pour exister; (3) doivent être considérées en tant que patrimoines, et non comme capital, au sens où il va être justifié, dans des périodes d'exploration où ils coûtent plus qu'ils ne rapportent, de faire l'effort de les préserver, en les faisant échapper au calcul économique traditionnel. En tant que faits stylisés, les patrimoines productifs sont des ressources immatérielles et matérielles et des institutions ou des organisations. Les ressources immatérielles sont des outils cognitifs partagés, des dispositifs de reconnaissance et de qualification de ressources libres présentant un intérêt pour un secteur donné (telles que des connaissances scientifiques) permettant des apprentissages entre utilisateurs et producteurs.

Il s'agit sur le plan matériel de dispositifs « localisés » qui permettent aux acteurs scientifiques et économiques de se rencontrer autour de laboratoires publics ou privés collectifs et d'activités de développement technologique (démonstrateurs ou unités pilotes avant industrialisation). Il s'agit ensuite d'institutions dédiées (Barrère, 2007 ) et d'outils institutionnels de constitution en communautés.

Le concept de patrimoine permet de lier passé, présent et futur en considérant le futur comme l'espace de projection de représentations héritées et formées dans 
le présent et le passé, qui vont guider les actions des acteurs dans le présent et se cristalliser dans des organisations et des institutions leur permettant de se reproduire dans le futur. La perspective ici mobilisée plaide en faveur d'une étude historicisée sur longue période des représentations et des engagements matériels de ce qui est aujourd'hui appelé «la stratégie de transition vers une bioéconomie». Resituer les success stories que les acteurs engagés dans des projets démonstratifs sont tenus de produire pour protéger leurs engagements sur des niches spécifiques permet d'identifier les stratégies destinées à capter les constructions des visions du futur ancrées dans des patrimoines productifs collectifs spécifiques.

\section{Une bioéconomie fondée sur la bioraffinerie?}

Après le premier choc pétrolier, les agro-industries et le secteur papetier remettent dans l'agenda des politiques publiques l'idée de productions industrielles à base renouvelable ( $c f$. quatrième partie). La pression des excédents agricoles et des surcapacités dans le monde papetier va les amener à préciser la gamme des produits atteignables à partir d'une théorisation de l'objet bioraffinerie et d'une stratégie de diffusion de son modèle dans des espaces variés (Cherubini et al., 2009; Nieddu et al., 2010).

Par analogie avec la raffinerie de pétrole, la bioraffinerie sera conçue comme l'unité fonctionnelle réalisant le craquage de matières premières d'origine végétale ou animale de natures et de qualités variées, afin de les ramener à des carburants liquides et à un petit nombre de grands intermédiaires chimiques permettant de préserver et de poursuivre la chimie du carbone. Comme la raffinerie de pétrole qui génère l'essentiel de la chimie de commodités à partir du vapocraquage en cinq grands intermédiaires ${ }^{4}$, la prospective menée par le ministère de l'Agriculture américaine (Werpy et Petersen, 2004) propose de cibler quatorze grands intermédiaires sélectionnés sur dire d'experts en croisant les espérances technologiques de substitution rapide et la taille des marchés (liste réduite à un top 10 par Bozell et Petersen, 2010). Bien que cette vision soit contestée en raison de son aspect réducteur ou de son manque d'attention à la soutenabilité, c'est bien celle-ci qui s'impose aujourd'hui dans le paysage de la bioéconomie (Morone et al., 2019).

Cette représentation dominante de l'avenir d'une chimie du végétal est fondée sur une chimie de commodités à partir de petites molécules légères comme les diacides, permettant de reformer des structures

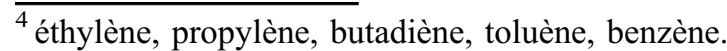

chimiques identiques à celles de produits issus du carbone fossile. Elle est débattue, car d'autres voies d'exploration du futur existent (Nieddu et al., 2014). Colonna et al. (2015) identifient ainsi deux autres paradigmes: la recherche de molécules d'origine renouvelable de structures différentes, mais fournissant les mêmes fonctions, et celle de nouvelles fonctionnalités atteignables grâce aux structures complexes que le vivant a su produire - et qu'il importe de ne pas détruire dans le processus de craquage. Néanmoins, la bioéconomie repose sur deux objectifs difficilement compatibles: augmenter l'usage de ressources naturelles grâce au progrès technique et assurer une utilisation durable de ces ressources grâce à une pression maîtrisée sur les écosystèmes (Levidow et al., 2013).

\section{La chemurgy, problématisation d'un sentier de développement}

\section{La chemurgy en tant que mouvement social en faveur de l'usage de ressources renouvelables}

Lors de son émergence, dans les années 1920, la chemurgy va réunir des personnages très contrastés. G.W. Carver, fils d'esclaves noirs à la date de naissance incertaine, cherche, dès les années 1890, à développer et à valoriser des productions agricoles nouvelles afin de sortir les fermiers noirs du «Sud profond» de leur pauvreté endémique. Sa mise à l'écart des aides fédérales à la recherche par le racisme des lois de l'Alabama ne l'a pas empêché de trouver plus d'une centaine d'applications non alimentaires du soja et des cacahuètes, de chercher à valoriser l'ensemble des déchets à la ferme, de trouver d'autres voies que celles des pesticides et des engrais chimiques et de protéger les sols de l'érosion et de la monoproduction. La «chimie créative» de Carver qui a précédé la Première Guerre mondiale (Abrams, 2008) laisse rêveur par sa proximité avec les usages attendus aujourd'hui: l'utilisation des coproduits de l'alimentation pour la fabrication de panneaux isolants, peintures, colorants, alcool industriel, matières plastiques de types divers, tapis, nattes et tissus, huiles, gommes et cires, etc. Il devient célèbre à la fin de la Première Guerre mondiale en proposant un procédé de production de caoutchouc à partir de patates douces.

Dès le milieu des années 1920, émerge un acteur inattendu: les sociétés de chemin de fer. Eprouvées par les crises de surproduction qui ont sévi après la Première Guerre mondiale, elles cherchent à planifier un développement régional, fondé sur la présence d'une agro-industrie «à la ferme» (Finlay, 1997), susceptible de leur ramener des affaires. L'improbable rencontre entre le fils d'esclaves agroécologiste avant la lettre et Henry Ford tient au fait que ce dernier cherchait à 
contourner le cartel de l'acier en produisant à partir de ressources renouvelables ses carrosseries et certaines pièces de ses automobiles. Il rêvait d'intégrer verticalement la totalité des matières premières, process et composants nécessaires à sa production automobile. Toujours est-il que, «In May 1935, Ford brought together over 300 leaders of agriculture, education, industry, and science in Dearborn, Michigan, for the first Dearborn Conference of Agriculture, Industry, and Science. Here the Farm Chemurgic Council was established, with Francis Garvan and the Chemical Foundation (a non-profit group dedicated to advancing the position of industrial chemistry) promising to support the group for the first year. » (Permeswaran, 2010, p. 97).

La Grande Dépression, si elle renforce l'intérêt pour la chemurgy, va surtout être un moment de conflit entre les isolationnistes et agrairiens et la présidence Roosevelt car les premiers prétendaient qu'un « libre» développement de la chemurgy était une alternative suffisante aux mesures du New Deal qu'ils jugeaient interventionnistes; néanmoins, ils finiront par accepter la création, en 1938, par Roosevelt de quatre laboratoires régionaux de l'USDA (le département de l'Agriculture des États-Unis) dédiés à la chemurgy. Ainsi, l'émergence de la chemurgy prend forme par la conjonction de trois phénomènes : (i) l'intérêt grandissant pour une nouvelle industrialisation de la chimie (Galambos et al., 2007), (ii) l'existence d'excédents de production agricoles et (iii) un débat politique entre isolationnistes et agrariens.

\section{Promesses de la chemurgy et constitution de réseaux d'acteurs}

C'est l'arrêt de projets d'envergure, la victoire du pétrole sur l'utilisation de ressources renouvelables et l'opposition à la chemurgy de leaders du monde agricole américain en faveur d'une spécialisation dans les produits agricoles de commodités plutôt qu'une stratégie de diversification vers de la biomasse non alimentaire qui permettent d'expliquer la fin de la chemurgy (Finlay, 2003). Deux éléments doivent être retenus pour comprendre les dynamiques contemporaines.

D'une part, la formation d'un espace méso-économique spécifique est un lieu de rencontre d'acteurs hétérogènes autour d'objets techniques. Au sein de cet espace, ils élaborent des réflexions sur l'ensemble des connaissances à produire, des ressources à assembler et des alliances politiques à construire pour soutenir l'effort industriel. Ils cherchent ainsi, à travers les épreuves de réalité, à rendre stable un régime particulier de production de connaissances et d'activités économiques.

D'autre part, les promesses technico-économiques nécessitent la production d'objets démonstratifs pour porter les récits de développement. Les différents produits proposés par Carver (souvent avec peu de succès commercial, mais toujours bien médiatisés au point qu'on en trouve trace dans de grands films de l'époque) ou, chez Ford, les prototypes de voitures dont les carrosseries sont réalisées en plastiques thermodurcissables à partir de renouvelables remplissent cette fonction. Il est donc intéressant de s'attacher à ces promesses en les projetant sur la situation actuelle (Tab. 1).

\section{Fin des années 1970: vers une bioindustrie?}

Ce deuxième moment de problématisation apparaît aux experts comme une crise plus profonde que la seule conjoncture des deux chocs pétroliers de 1973 et 1979. C'est ce qui ressort des constats faits dans les numéros spéciaux de la Revue d'économie industrielle et de Futuribles consacrés à la bioindustrie qui paraissent au début des années 1980. Ce dernier fait écho à la position de la Communauté économique européenne sur le sujet, issue d'un programme de la direction générale XII (Science, recherche et développement).

\section{Trois constats à l'origine de la réflexion sur le développement des bioindustries}

Le premier constat est le net ralentissement du rythme d'innovation dans la chimie par rapport aux années 1950-1960, attesté par des études économétriques ultérieures comme celle d'Achilladelis et al. (1990). Le deuxième constat est la saturation des grands marchés construits grâce à des monopoles de droits de propriété et à la stratégie dite de l'ultimate plant, théorisée par la société Du Pont de Nemours, qui veut que les investissements de productivité permettent d'abaisser drastiquement les coÛts de production afin de dissuader l'entrée de concurrents. Le revers d'une telle stratégie est la constitution de rigidités structurelles peu à même de faire face à l'instabilité nouvelle due à la saturation des marchés et à l'instabilité des prix amont et aval. Le troisième constat est que les États (et la Commission européenne), associés avec les grandes entreprises de la chimie, concernés par la crise des grandes unités de production chimique, vont concevoir des états de l'art et des hypothèses prospectives sur les domaines susceptibles de relancer la croissance et l'innovation (Van Laer, 2010). Ces états de l'art sont constitutifs du travail que les acteurs réalisent autour des promesses technologiques, ils sont le reflet de la projection de patrimoines productifs collectifs spécifiques.

\section{Chimie et «bioindustries classiques » au cœur de la réflexion française}

Le numéro de la Revue d'économie industrielle eut droit à une introduction du tout récent ministre de la 
Tab 1. Tableau comparatif des promesses technico-économiques de la chemurgy et de la bioéconomie.

\begin{tabular}{|c|c|c|}
\hline Période & Matières premières et produits visés & Produits toujours visés actuellement \\
\hline $1920-1934$ & $\begin{array}{l}\text { - Lait de soja } \rightarrow \text { peintures, lubrifiants, plastiques } \\
\text { d'automobiles } \\
\text { - Épi de maïs, déchets de pin, patate douce, } \\
\text { chanvre et grains divers comme matières } \\
\text { premières } \\
\text { - Alcool naturel (éthanol) pour l'énergie et } \\
\text { mélanges essence/éthanol } \\
\text { - Cellulose, amidon, lignine, fructose (= sucres et } \\
\text { fibres) }\end{array}$ & $\begin{array}{l}\text { - Huiles de soja pour la lubrification (Biopress par } \\
\text { exemple) } \\
\text { - Déchets de pin comme les écorces pour la } \\
\text { production de mousses isolantes, matériaux à base } \\
\text { de chanvre, blé pour la production de PHA } \\
\text { (plastique biodégradable à forte valeur ajoutée) } \\
\text { - Taux d'incorporation déjà existant, généralisation } \\
\text { de la production de biocarburants } \\
\text { - Lignine (matériaux vs. énergie), amidon (matériaux } \\
\text { vs. chimie des molécules), cellulose dans les } \\
\text { matériaux et l'hygiène (dentifrice par exemple) }\end{array}$ \\
\hline 1935-1939 & $\begin{array}{l}\text { - Utilisation des déchets de scieries pour la } \\
\text { production de matériaux et plastiques } \\
\text { - Sucre de betterave, déchets d'artichauts, déchets } \\
\text { de ferme } \rightarrow \text { butanol, acétone } \\
\text { - Chanvre, lin, riz } \rightarrow \text { papier } \\
\text { - Riz } \rightarrow \text { furfural et glycérine amidons de patate } \\
\text { douce } \\
\text { - Sucre de canne, sorgho, patates douces } \\
\rightarrow \text { éthanol } \\
\text { - Pins, tung } \rightarrow \text { journaux } \\
\text { - Cellulose } \rightarrow \text { fibres synthétiques, matériaux pour } \\
\text { l'automobile }\end{array}$ & $\begin{array}{l}\text { - Réutilisation des déchets de scieries pour } \\
\text { matériaux et plus uniquement énergies } \\
\text { - Butanol biosourcé } \\
\text { - Furfural } \\
\text { - Généralisation de l'éthanol } \\
\text { - Techniques d'extraction de celluloses }\end{array}$ \\
\hline $1939-1945$ & $\begin{array}{l}\text { - Sources américaines de caoutchouc } \\
\text { - Molécules pour la pharmacie } \\
\text { - Blé } \rightarrow \text { adhésifs } \\
\text { - Canne à sucre } \rightarrow \text { graisse } \\
\text { - Caséine } \rightarrow \text { vêtements, fibres } \\
\text { - Fermentation pour la production d'antibiotiques }\end{array}$ & $\begin{array}{l}\text { - Réintroduction du caoutchouc d'origine naturelle } \\
\text { (Michelin) } \\
\text { - Cartons et adhésifs par l'amidon de blé (roquette) } \\
\text { - Techniques fermentaires }\end{array}$ \\
\hline 1945-1972 & $\begin{array}{l}\text { - Huiles végétales } \rightarrow \text { lécithine, glycérine, } \\
\text { plastiques, adhésifs, retardateurs } \\
\text { d'inflammabilité } \\
\text { - Fermentation utilisant l'acide lactique, citrique, } \\
\text { gluconique }\end{array}$ & $\begin{array}{l}\text { - Polyuréthanes à partir des huiles végétales } \\
\text { - PLA à partir de fermentation d'acide lactique }\end{array}$ \\
\hline
\end{tabular}

Dans chacune des colonnes, il est question soit de produits effectivement produits, soit de programmes de R\&D (réalisation: N. Béfort, M. Nieddu, d'après Béfort, 2016).

Recherche d'alors, Jean-Pierre Chevènement, intitulée : «La France à l'ère bioindustrielle», qui dessine les enjeux en termes d'exploitation industrielle de la révolution scientifique du génie génétique. Néanmoins, comme plusieurs auteurs de ce numéro spécial, il souligne l'existence de bioindustries «classiques». Les bioindustries et la chimie «classiques » sont au cœur du développement d'une science de l'ingénieur des processus en continu, condition essentielle pour réaliser des gains de productivité dans de grandes raffineries (Danielou et Broun, 1981). Au-delà des innovations de produits introduites par le génie génétique, c'est la capacité à proposer des réactions catalytiques qui est la qualité la plus appréciée des biotechnologies. En effet, celles-ci permettent d'envisager une amélioration de l'efficacité des procédés. Or, il apparaît que les industriels n'adopteront les biotechnologies que lorsqu'elles réussiront à contester le process existant : «Il importe dans tous les cas que le choix de la filière soit économiquement fondé : on ne choisit pas la voie biologique parce que c'est prestigieux, à la mode, écologique. On le fait lorsque la comparaison économique est en sa faveur. » (Penasse, 1981, p. 37). Entre les deux situations polaires (domination du process chimique classique versus celle d'un process 
biotechnologique), Penasse voit exister un ensemble de voies technologiques qu'il qualifie d'hémisynthèse (couplage ou utilisation en cascade de réactions chimiques et biotech) créant un ensemble de nouvelles opportunités économiques.

Néanmoins, la disparition des procédés thermochimiques du paysage des bioindustries au profit des procédés biotechnologiques n'est pas envisagée. Pour se repérer dans l'espace des trajectoires concurrentes, les acteurs ont produit des schémas analytiques des filières principales. Ceux-ci permettent de constater que le paysage actuel est déjà cartographié en 1981. Par exemple, Chesnais (1981) recense les matières premières évoquées aujourd'hui, des schistes bitumineux à la biomasse ou aux déchets. La biomasse est transformée dans des procédés qui vont soit vers les gaz de synthèse (thermochimie) imitant la chimie du pétrole, soit vers des procédés de fermentation rebouclant sur celle-ci à partir de l'éthanol ou des diacides. Fondamentalement, ce sont donc les mêmes chaînes carbonées que celles issues du «roi pétrole» qui sont visées. D'où une confusion entretenue entre bioindustries mobilisant un process biotechnologique et celles mobilisant des biomasses, quel que soit le procédé.

L'exercice de problématisation se concentre alors sur les ruptures schumpétériennes au sein des agro-industries existantes et fait apparaître deux éléments qui nous semblent structurants aujourd'hui. D'une part, l'innovation entraîne surtout le développement d'une hyperconcurrence entre matières premières biosourcées dès lors que les avancées scientifiques des biotechnologies peuvent devenir des procédés industriels continus (Zitt, 1983 , p. 42). D'autre part, l'innovation biotechnologique $^{5}$ permet de reconfigurer la chaîne de valeur afin d'obtenir le contrôle de la " global supply chain » à partir des produits intermédiaires: «Enfin cette innovation mixte procédé-produit est significative de tendances lourdes d'évolution technologique dans les bioindustries: le développement des marchés de produits intermédiaires affranchis d'une source agricole unique, et l'émergence d'un nouvel opérateur technique, les «enzymes immobilisées », dont la production d'isoglucose est à ce jour la plus importante application industrielle » (Zitt, 1983, p. 42).

De ce point de vue, la variable-clé du changement est bien économique. Chesnais (1981, p. 222) rappelle que « théoriquement, pratiquement n'importe quel produit de la chimie industrielle organique peut être produit par un

\footnotetext{
${ }^{5}$ Précisons qu'il s'agit de biotechnologies industrielles qu'on qualifierait aujourd'hui de classiques au sens où il s'agit de préparer des enzymes à des usages en conditions industrielles et non des «nouvelles biotech» fondées sur les manipulations en génie génétique.
}

processus biologique $»$. Les recherches en biotech sont donc orientées dans un sens précis: venir contester chaque process chimique ou thermochimique existant afin de se demander s'il est possible d'envisager une substitution de ces process, à produit ou fonction finale (pouvoir sucrant, par exemple) identique. Ce type d'orientation fondée sur une vision du futur de la chimie hybridée aux biotechnologies génère donc bien un régime particulier de production de connaissances - et des activités économiques en découlant (Cohendet et al., 1987). Celui-ci est dédié à l'enrichissement de patrimoines productifs collectifs, eux aussi spécifiques, sans que les patrimoines de la thermochimie visés par la substitution ne disparaissent.

\section{Quelle(s) structure(s) d'une chimie fondée sur les bioindustries?}

On peut ainsi passer à une relecture du dossier de Futuribles largement inspiré du travail de l'OCDE en 1978-1979 et du programme FAST EUR7767 de la Commission européenne (direction générale XII) regroupant chercheurs et grandes firmes de la chimie sur les perspectives de la chimie en Europe (bien connus grâce aux publications du Bureau d'économie théorique et appliquée [BETA] de l'Université de Strasbourg; Ancori et Cohendet, 1984). Il identifie quatre thèmes principaux autour desquels il propose d'articuler la politique d'innovation à l'échelle européenne: la chimie des petites molécules ${ }^{6}$ (liée au renouvellement de la carbochimie), la chimie des sucres, la chimie des matériaux nouveaux et la chimie de la fonction.

Or, cette façon d'organiser les thématiques laisserait penser que la bioindustrie est réservée à la chimie des sucres, ce qui n'est pas le cas, comme nous allons le montrer. Les enjeux de la chimie des petites molécules sont discutés, comme ceux d'une renaissance de la chimie du charbon. Son cœur réside dans le fait que l'on pouvait imaginer de substituer aux intermédiaires issus de la pétrochimie un intermédiaire issu d'une gazéification des chaînes carbonées du charbon, le méthanol, sur lequel il est possible de fonder un ensemble d'espérances technologiques. Tout comme les cinq intermédiaires de base de la pétrochimie, «l'aval de la chimie du méthanol est très riche » (Cohendet, 1982, p. 17) car il permet de reformer des carburants et de l'acide acétique, qui sert de base notamment à des plastiques (le PET) ou des solvants, des peintures et des vernis (encadré 1).

\footnotetext{
${ }^{6}$ La « chimie des petites molécules » désigne la chimie liée à la production de molécules chimiques à faible nombre (un à trois) d'atomes de carbone alors que les sucres en contiennent plus. Ce mode de classement est structurant pour la réflexion sur la transition d'une chimie durable. Pour un exposé détaillé, cf. Nieddu et al., 2014 dans Nature Sciences Sociétés.
} 


\section{Encadré 1. L'acide acétique à partir d'éthylène}

L'acide acétique produit à partir d'éthylène pétrolier, de méthanol de la chimie du charbon, ou par voie biologique (le bon vieux vinaigre...) peut servir de:

- solvant : miscible à l'eau et à divers solvants organiques tels l'éthanol, l'oxyde de diéthyle, le glycérol, mais insoluble dans le sulfure de carbone, c'est aussi un bon solvant des gommes-résines, du phosphore, du soufre et d'acides halogénés ;

- production d'anhydride acétique, d'acétate de cellulose, d'acétate de vinyle monomère, et d'autres acétates, ainsi que de médicaments, pesticides, colorants, produits de l'industrie de la photographie ;

- alimentation (production de vinaigres de fruit...), additif alimentaire;

- textiles;

- agent de nettoyage (par exemple de de semi-conducteurs);

- coagulant (du latex naturel);

- bactériostatique (en solution);

- dans la fabrication de plastiques tels le polytéréphtalate d'éthylène (PET) ou l'acétate de cellulose, utile à la production d'acétate de vinyle (peintures, adhésifs) et de solvants organiques;

- additif dans les produits dérivés du tabac (arôme).

(source : N. Béfort, M. Nieddu, d'après Cheung et al., 2011).

Tab 2. Les patrimoines productifs collectifs de la chemurgy, de la bioindustrie et de la bioéconomie.

\begin{tabular}{|c|c|c|c|}
\hline & Chemurgy & Bioindustrie & Bioéconomie \\
\hline $\begin{array}{l}\text { Ressources matérielles } \\
\text { et immatérielles }\end{array}$ & $\begin{array}{l}\text { - Coproduits de } \\
\text { l'alimentation et de } \\
\text { l'agriculture } \\
\text { - Excédents de production } \\
\text { agricole } \\
\text { - Avancées dans } \\
\text { l'industrialisation de la } \\
\text { chimie } \\
\text { - Laboratoires régionaux } \\
\text { pour la chemurgy }\end{array}$ & $\begin{array}{l}\text { - Financement de projets } \\
\text { prospectifs par les pouvoirs } \\
\text { publics } \\
\text { - Opportunités offertes par } \\
\text { l'apparition de nouveaux } \\
\text { procédés }\end{array}$ & $\begin{array}{l}\text { - Bases de connaissances } \\
\text { hétérogènes } \\
\text { - Financements de projets } \\
\text { - Biomasse issue de } \\
\text { l'agriculture agro- } \\
\text { industrielle }\end{array}$ \\
\hline $\begin{array}{l}\text { Dispositifs de qualification } \\
\text { et de reconnaissance }\end{array}$ & $\begin{array}{l}\text { - Formation d'un bloc } \\
\text { social liant isolationnistes } \\
\text { et agrariens } \\
\text { - Farm Chemurgic Council } \\
\text { - Intégration des projets de } \\
\text { chemurgy dans de } \\
\text { grandes firmes (Ford) }\end{array}$ & $\begin{array}{l}\text { - États de l'art qualifiant les } \\
\text { technologies } \\
\text { - Possibilité de concurrencer } \\
\text { un procédé existant }\end{array}$ & $\begin{array}{l}\text { - Définitions de la } \\
\text { bioéconomie } \\
\text { - Financements de projets } \\
\text { visant des valorisations } \\
\text { non alimentaires } \\
\text { - L'utilisation de } \\
\text { ressources renouvelables, } \\
\text { si possible de façon } \\
\text { soutenable }\end{array}$ \\
\hline $\begin{array}{l}\text { Dispositifs localisés } \\
\text { de coordination }\end{array}$ & $\begin{array}{l}\text { - Chemurgic Council } \\
\text { - Développement d'unités } \\
\text { de production } \\
\text { - Démonstration de } \\
\text { produits emblématiques }\end{array}$ & $\begin{array}{l}\text { - Groupes de réflexion et de } \\
\text { prospective } \\
\text { - Chimie des petites } \\
\text { molécules } \\
\text { - Produits emblématiques } \\
\text { - Production centralisée } \\
\text { versus décentralisée }\end{array}$ & $\begin{array}{l}\text { - Bioraffinerie et pilotes } \\
\text { industriels } \\
\text { - Molécules plateformes } \\
\text { versus nouvelles } \\
\text { fonctionnalités } \\
\text { - Promesse de transition } \\
\text { portée par la } \\
\text { bioéconomie et ses } \\
\text { produits }\end{array}$ \\
\hline
\end{tabular}


Or, le méthanol peut être obtenu à partir de biogaz

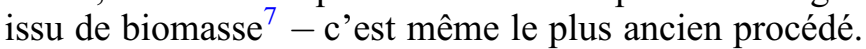
De même, les bioindustries au sens strict (le travail au niveau de la cellule par un procédé biotech) peuvent produire des petites molécules qui se substituent aux grands intermédiaires chimiques de la pétrochimie. Les promesses technico-économiques de la bioraffinerie (au sens d'une imitation de la raffinerie de pétrole) viennent donc de loin. Cette voie est documentée dans un article s'appuyant sur l'exemple du méthanol et utilisant explicitement l'appellation de bioraffinerie dès 1980 .

Des voies biosourcées de chimie des matériaux et de chimie de fonction sont également analysées. C'est donc dans les quatre thèmes du rapport sur les perspectives de la chimie en Europe qu'il faut rechercher les traces d'une problématisation de la bioindustrie et non dans un seul, celui de la chimie des sucres. Ce constat n'est pas surprenant si l'on suit les travaux de Colombo qui va être le fondateur de Novamont, aujourd'hui une entreprise phare de la reconversion de la chimie italienne vers les renouvelables (Béfort et Nieddu, 2017).

\section{Conclusion : quels enseignements pour l'étude de la bioéconomie dans sa période actuelle?}

Cette contribution s'est intéressée à l'étude de deux moments de problématisation du futur. Dans ces deux moments particuliers, des acteurs ont cherché à construire des visions de l'usage de ressources renouvelables. Le tableau 2 propose une mise en parallèle des caractéristiques de la chemurgy, de la bioindustrie des années 1980 et de ce qui est qualifié aujourd'hui de bioéconomie. Il montre la grande permanence des ressources mobilisées, des dispositifs de qualification et de coordination et des problématiques productives collectives sous-jacentes. Nous avons pointé le rôle dominant de la chimie des petites molécules dans les visions du futur des bioindustries, résultat que l'on a retrouvé dans la bioéconomie actuelle (Nieddu et al., 2014). En effet, cette chimie s'est d'abord imposée dans celle des matières fossiles, associée à des unités de production de grande taille, fournissant de grands intermédiaires à la chimie de base. Ce modèle va orienter de façon significative la recherche en bioraffinerie sur les petites molécules du renouvelable connues dès l'époque de la chemurgy.

\section{Références}

Abrams D., 2008. George Washington Carver: scientist and educator, New York, Chelsea House.

\footnotetext{
${ }^{7}$ Pour une revue de littérature, voir, par exemple, Sawatdeenarunat et al. (2016).
}

Achilladelis B., Schwarzkopf A., Cines M., 1990. The dynamics of technological innovation: the case of the chemical industry, Research Policy, 19, 1, 1-34, http://dx. doi.org/10.1016/0048-7333(90)90032-2.

Ancori B., Cohendet P. (Eds), 1984. La chimie en Europe : innovations, mutations et perspectives, Paris, Économica.

Barrère C., 2007. Vers une théorie économique substantiviste du patrimoine, Économie Appliquée, 3, 7-30.

Beckert J., 2013. Capitalism as a system of expectations: toward a sociological microfoundation of political economy, Politics \& Society, http://dx.doi.org/10.1177/ 0032329213493750.

Beckert J., 2016. Imagined futures: fictional expectations and capitalist dynamics, Cambridge, Harvard University Press.

Béfort N., 2016. Pour une mésoéconomie de l'émergence de la bioéconomie : représentations, patrimoines productifs collectifs et stratégies d'acteurs dans la régulation d'une chimie doublement verte. Thèse de doctorat en sciences économiques, Reims, Université de Reims ChampagneArdenne.

Béfort N., Nieddu M., 2017. De la variété des ancrages territoriaux des firmes en Chimie Doublement Verte, Géographie, Économie, Société, 19, 1, 133-152.

Borup M., Brown N., Konrad K., Van Lente H., 2006. The sociology of expectations in science and technology, Technology Analysis \& Strategic Management, 18, 3-4, 285-298, http://dx.doi.org/10.1080/09537320600777002.

Boyer R., 2018. Expectations, narratives, and socio-economic regimes, in Beckert J., Bronk R. (Eds), Uncertain futures. Imaginaries, narratives, and calculation in the economy, Oxford, Oxford University Press, 39-61, http://dx.doi.org/ 10.1093/oso/9780198820802.003.0002.

Bozell J.J., Petersen G.R., 2010. Technology development for the production of biobased products from biorefinery carbohydrates-the US Department of Energy's “Top 10" revisited, Green Chemistry, 12, 539-554, http://dx.doi.org/ 10.1039/B922014C.

Cherubini F., Jungmeier G., Wellisch M., Willke T., Skiadas I., Van Ree R., de Jong E., 2009. Toward a common classification approach for biorefinery systems, Biofuels, Bioproducts and Biorefining, 3, 5, 534-546, http://dx.doi. org/10.1002/bbb.172.

Chesnais F., 1981. Biotechnologie et modifications des structures de l'industrie chimique: quelques points de repère, Revue d'économie industrielle, 18, 218-230, http:// dx.doi.org/10.3406/rei.1981.1112.

Cheung H., Tanke R.S., Torrence G.P., 2011. Acetic Acid, in Elvers B. (Ed.), Ullmann's encyclopedia of industrial chemistry, Weinheim, Wiley-VCH, https://doi.org/10.1002/ 14356007.a01_045.pub2.

Cohendet P., 1982. L'industrie chimique européenne et la crise, la nécessité des mutations technologiques, Futuribles, 60, 13-31.

Cohendet P., Ledoux M.-J., Zuscovitch E., 1987. Les matériaux nouveaux : dynamique économique et stratégie européenne, Paris, Économica.

Colonna P., Tayeb J., Valceschini E., 2015. Les nouveaux usages de la biomasse, in Le Déméter 2015, Paris, Club Déméter éditeur, 275-305. 
Danielou G., Broun G., 1981. Bioindustrie: de la tradition artisanale à la pratique industrielle, Revue d'économie industrielle, 18, 14-29, http://dx.doi.org/10.3406/ rei.1981.1088.

European Commission, 2012. Communication from the Commission to the European Parliament, the Council, in Innovating for sustainable growth: a bioeconomy for Europe, Luxembourg, Publications Office of the European Union, 9-17.

Finlay M.R., 1997. the failure of chemurgy in the depressionera south: the case of Jesse F. Jackson and the Central of Georgia Railroad, The Georgia Historical Quarterly, 81, 1, 78-102.

Finlay M.R., 2003. Old efforts at new uses: a brief history of chemurgy and the American search for biobased materials, Journal of Industrial Ecology, 7, 3-4, 33-46. https:// on linelibrary.wiley.com/doi/abs/10.1162/ 108819803323059389.

Galambos L., Hikino T., Zamagni V. (Eds), 2007. The global chemical industry in the age of the petrochemical revolution, Cambridge, Cambridge University Press.

Joly P.-B., 2010. On the economics of techno-scientific promises, in Akrich M., Barthe Y., Muniesa F. (Eds), Débordements. Mélanges offerts à Michel Callon, Paris, Presse des Mines, 203-221, http://books.openedition.org/ pressesmines $/ 747$.

Jullien B., Smith A., 2012. Le gouvernement d'une industrie. Vers une économie politique institutionnaliste renouvelée, Gouvernement et action publique, 1, 103-123, http://dx.doi. org/10.3917/gap.121.0103.

Langeveld H., Meeusen M., Sanders J. (Eds), 2010. The biobased economy: biofuels, materials and chemicals in the post-oil era, London/Washington (DC), Earthscan.

Levidow L., Birch K., Papaioannou T., 2013. Divergent paradigms of European agro-food innovation: the knowledge-based bio-economy (KBBE) as an R\&D agenda, Science, Technology, \& Human Values, 38, 1, 94-125, http:// dx.doi.org/10.1177/0162243912438143.

Lokko Y., Heijde M., Schebesta K., Scholtès P., Van Montagu M., Giacca M., 2018. Biotechnology and the bioeconomytowards inclusive and sustainable industrial development, New Biotechnology, 40, Part A, 5-10, http://dx.doi.org/ 10.1016/j.nbt.2017.06.005.

Morone P., Falcone P.M., Lopolito A., 2019. How to promote a new and sustainable food consumption model: a fuzzy cognitive map study, Journal of Cleaner Production, 208, 563-574. http://dx.doi.org/10.1016/j.jclepro.2018.10.075.

Nieddu M., 2013. Évaluation et changement technologique : réflexions sur le cas des agromatériaux, in Vivien F.-D., Lepart J., Marty P. (Eds), L'évaluation de la durabilité, Paris, Quæ, 175-190, http://dx.doi.org/10.3917/quae.vivie.2013.01.0175.
Nieddu M., Garnier E., Bliard C., 2010. L'émergence d'une chimie doublement verte, Revue d'économie industrielle, 132, 4, 53-84, http://dx.doi.org/10.4000/rei.4355.

Nieddu M., Garnier E., Bliard C., 2014. Patrimoines productifs collectifs versus exploration/exploitation. Le cas de la bioraffinerie, Revue économique, 65, 6, 957-987, http://dx. doi.org/10.3917/reco.pr2.0031.

Nieddu M., Vivien F.-D., Garnier E., Bliard C., 2014. Existe-til réellement un nouveau paradigme de la chimie verte? Natures Sciences Sociétés, 22, 2, 103-113, https://doi.org/ $10.1051 / \mathrm{nss} / 2014022$.

Penasse L., 1981. Perspectives et contraintes de la bioindustrie, Revue d'économie industrielle, 18, 30-37, http://dx.doi.org/ 10.3406/rei.1981.1089.

Permeswaran P., 2010. Chemurgy: using science innovatively to save American agriculture from overproduction, The History Teacher, 44, 1, 95-125.

Pursell C.W., 1969. The Farm Chemurgic Council and the United States Department of Agriculture, 1935-1939, Isis, 60, 3, 307-317, http://dx.doi.org/10.1086/350500.

Sawatdeenarunat C., Nguyen D., Surendra K.C., Shrestha S., Rajendran K., Oechsner H., Xie L., Khanal S.K., 2016. Anaerobic biorefinery: current status, challenges, and opportunities, Bioresource Technology, 215, 304-313, http://dx.doi.org/10.1016/j.biortech.2016.03.074.

Shurtleff W., Aoyagi A. (Eds), 2011. Henry Ford and his researchers: history of their work with soybeans, soyfoods and chemurgy (1928-2011): extensively annotated bibliography and sourcebook, Lafayette (CA), Soyinfo Center, www.soyinfocenter.com/pdf/145/Ford.PDF.

Staffas L., Gustavsson M., McCormick K., 2013. Strategies and policies for the bioeconomy and bio-based economy: an analysis of official national approaches, Sustainability, 5, 6, 2751-2769, http://dx.doi.org/10.3390/su5062751.

Van Laer A., 2010. Vers une politique de recherche commune : du silence du traité CEE au titre de l'Acte Unique, in Bouneau C., Burigana D., Varsori A. (Eds),Trends in technological innovation and the European construction: the emerging of enduring dynamics?, Brussels, Peter Lang, 79-100.

Vivien F.-D., Nieddu M., Befort N., Debref R., Giampietro M., 2019. The hijacking of the bioeconomy, Ecological Economics, 159, 189-197, http://dx.doi.org/10.1016/j.eco lecon.2019.01.027.

Werpy T., Petersen G., 2004. Top value added chemicals from biomass, vol. 1, US Department of Energy, p. 76, http://dx. doi.org/10.2172/15008859.

White H.C., Godart F., Corona V., 2008. Produire en contexte d'incertitude. La construction des identités et des liens sociaux dans les marchés, Sciences de la Société, 73, 17-40.

Zitt M., 1983. Un cas d'innovation: l'isoglucose, Économie rurale, 158, 42-50. http://dx.doi.org/10.3406/ecoru.1983.3010.

Citation de l'article : Béfort N., Nieddu M. Bioéconomie : un retour historique sur deux problématisations de l'usage des ressources renouvelables. Nat. Sci. Soc. 28, 3-4, 216-225. 